\title{
Photodynamic therapy for actinic keratosis: a trend towards a decrease in irradiance without loss of efficacy for a better tolerability
}

\author{
Anne-Sophie Vignion-Dewalle ${ }^{\mathrm{a}}$, Claire Vicentini ${ }^{\mathrm{a}, \mathrm{b}}$, Gregory Baert ${ }^{\mathrm{a}}$, Elise Thecua ${ }^{\mathrm{a}}$, Fabienne Lecomte ${ }^{\mathrm{a}}$, \\ Laurent Mortier ${ }^{\mathrm{a}, \mathrm{b}}$, Serge Mordon ${ }^{\mathrm{a}}$
}

${ }^{a}$ Univ. Lille, INSERM, CHU Lille, U1189 - ONCO-THAI - Image Assisted Laser Therapy for Oncology, F59000 Lille, France; 'Department of Dermatology, CHU Lille, F-59000 Lille, France

\begin{abstract}
Photodynamic therapy (PDT) is an established treatment for actinic keratosis (AK). The conventional approved PDT protocol in Europe (C-PDT) involves red-light photoactivation at irradiances higher than $60 \mathrm{~mW} / \mathrm{cm}^{2}$, making the treatment painful. Several clinical studies have reported similar efficacy and better tolerability when using red-light photoactivation at lower irradiances.

The aim of the study was to investigate whether there is a minimum irradiance threshold for red-light photoactivation above which there is no further improvement in efficacy.

A photodiode sensor connected to a power meter was used to measure the irradiance delivered to 114 AKs on the scalp and forehead of 19 patients during C-PDT using the Aktilite CL 128 (Galderma SA, Switzerland). The widely ranging measured irradiances, resulting from the heterogeneous photoactivation over the treatment area provided by the Aktilite CL 128, were cross-referenced with the clinically evaluated complete responses (CR) at 3 months.

The $66 \mathrm{AKs}$ in CR at 3 months received an average irradiance of $30.9 \mathrm{~mW} / \mathrm{cm}^{2}$ (standard deviation: $16.7 \mathrm{~mW} / \mathrm{cm}^{2}$ ) compared to $33.3 \mathrm{~mW} / \mathrm{cm}^{2}$ (standard deviation: $17.9 \mathrm{~mW} / \mathrm{cm}^{2}$ ) for the $48 \mathrm{AKs}$ in incomplete response. No significant effect of the irradiance on the $\mathrm{CR}$ at 3 months was found (odds ratio for a $6 \mathrm{~mW} / \mathrm{cm}^{2}$-unit change, $0.96 ; 95 \%$ confidence interval, 0.83 to $1.10 ; \mathrm{p}=0.53$ ). No minimum irradiance threshold could therefore be determined in the considered irradiance range.
\end{abstract}

A red-light device enabling homogeneous irradiation at a lower irradiance than the Aktilite CL 128 may therefore provide similar efficacy and higher treatment tolerability than C-PDT.

Keywords: Photodynamic therapy; actinic keratosis; Aktilite CL128; irradiance; complete response

\section{INTRODUCTION}

Photodynamic therapy (PDT), which is based on the photoactivation of a photosensitizer by light, is an effective, noninvasive treatment for actinic keratosis (AK) [1, 2]. In Europe, the approved protocols for PDT of AK involve redlight or daylight photoactivation. Red-light photoactivation with the Aktilite CL 128 (Galderma SA, Switzerland) using a total light dose of $37 \mathrm{~J} / \mathrm{cm}^{2}$ after $3 \mathrm{~h}$ of incubation with methyl aminolevulinate (MAL) is a conventional PDT protocol, usually referred to as C-PDT [3, 4]. Due to the irradiances higher than $60 \mathrm{~mW} / \mathrm{cm}^{2}$ generated by the lamp, C-PDT is known to be painful [5]. Very recently, red-light photoactivation at irradiances of $15.4 \mathrm{~mW} / \mathrm{cm}^{2}$ [6], $12.3 \mathrm{~mW} / \mathrm{cm}^{2}$ [7] and $1.3 \mathrm{~mW} / \mathrm{cm}^{2[8]}$ respectively, has demonstrated similar efficacy and strong pain reduction compared to C-PDT.

These results raise the question of whether there is a minimum irradiance threshold for red-light photoactivation above which there is no further improvement in efficacy. This threshold would ensure a maximum tolerability without loss of efficacy.

To address this question, we have used data collected within an above-referenced clinical trial ${ }^{[7]}$. This data consists of the irradiances measured during photoactivation with the Aktilite CL 128 (Galderma SA, Switzerland) in 114 AKs on the forehead and scalp of 19 patients. Due to the heterogeneous photoactivation over the treatment area provided by the Aktilite CL 128, a large range of irradiances was ensured. The clinically evaluated complete responses (CR) at 3 months 
for the 114 AKs were also part of this data. From this data, we first determined whether the irradiance has a significant effect on the CR at 3 months. In case a significant effect was found, we then identified the minimum irradiance threshold for red-light photoactivation above which there is no further improvement in efficacy.

\section{PATIENTS, MATERIALS AND METHODS}

\subsection{Study design}

This was an ancillary study of the Flexitheralight clinical trial registered as NCT03076918 at www.clinicaltrials.gov. The Flexitheralight clinical trial that was conducted in the Department of Dermatology of Lille University Hospital aimed to assess the non-inferiority of a new protocol involving irradiation with a light-emitting, fabric-based device compared to C-PDT for PDT of AK [7, 9].

\subsection{Patients}

Subjects for the present study were recruited among patients enrolled in the Flexitheralight clinical trial from October 2014 to October 2015. The patients had to be aged 18 years or older, with two areas of five to seven nonpigmented grade I (thin) or grade II (moderately thick) AKs on the forehead and/or scalp. For each patient, one area was randomized to receive PDT using the new protocol and the other was randomized to be treated with C-PDT. Only the lesions in the CPDT area were included in the present study.

All patients gave written informed consent before entering the study.

\subsection{Materials}

The irradiances were measured using an OPHIR PD300 photodiode sensor connected to an OPHIR LaserStar power meter (Ophir Photonics, Jerusalem, Israel) (Figure 1).

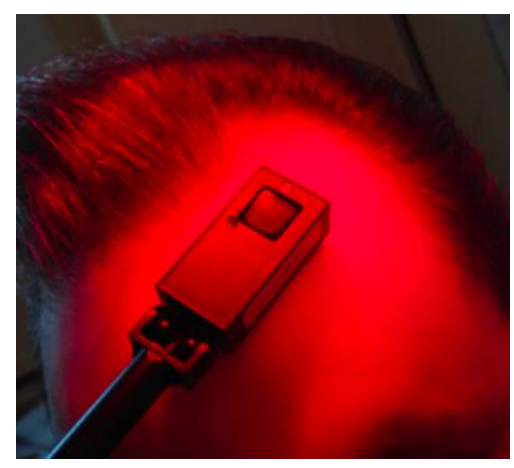

Figure 1. Irradiance measurement with the PD300 photodiode sensor.

\subsection{Treatment}

Demographic and clinical characteristics of patients were recorded at the screening visit. On the day of treatment, AKs on the forehead and/or scalp were counted, graded, photographed and divided into two areas of five to seven nonpigmented grade I (thin) or grade II (moderately thick) AKs (the two areas were similar with respect to number and grades of lesions). Randomization was then performed.

The area randomized to receive C-PDT was prepared by superficial curettage, and approximately 1 to $2 \mathrm{~g}$ of MAL cream (Metvixia, Galderma) was applied under occlusive dressing and then covered with aluminum foil. After three hours of 
incubation, MAL cream was removed with a saline solution, and the Aktilite CL 128 was placed between 8 and $10 \mathrm{~cm}$ from the treatment area and programmed to deliver $37 \mathrm{~J} / \mathrm{cm}^{2}$ in 10 minutes.

During photoactivation with the Aktilite CL 128, the OPHIR PD300 photodiode sensor was applied over each lesion, with its face towards the Aktilite CL 128, and the displayed irradiances were recorded (one irradiance per lesion per patient). All the measurements were made, according to a standardized procedure, by a single operator to avoid interoperator variability.

A clinical evaluation of the CR at 3 months was performed.

\subsection{Data analysis}

Continuous variables are expressed as the mean, standard deviation (SD) and range, and categorical variables are expressed as a frequency and percentage.

The effect of the irradiance on the CR at 3 months was assessed using a generalized linear mixed model with a binomial distribution and logit link function. The irradiance and the grade were included as fixed effects. The patient was considered as a random effect to account for repeated measures from individual patients. Effect size was estimated by odds ratio (OR) and its $95 \%$ confidence interval (CI). The significance level was set at a two-sided alpha level of 0.05 .

Statistical analyses were performed using SAS software version 9.4 (SAS Institute Inc., Cary, North Carolina, USA).

\section{RESULTS}

\subsection{Patients study}

Nineteen male patients were included in the study (Table 1). In these patients, a total of 114 AKs received C-PDT and was considered in the present study (Table 2).

Table 1. Patients characteristics on the day of treatment.

\begin{tabular}{|l|l|}
\hline \multicolumn{1}{|c|}{ Patients characteristics } & Values \\
\hline Age (years) & \\
Mean \pm standard deviation (SD) & $70.7 \pm 11.0$ \\
Range & $51-87$ \\
\hline Fitzpatrick skin phototype (\%) & \\
I & $1(5.3)$ \\
II & $17(89.4)$ \\
III & $1(5.3)$ \\
\hline
\end{tabular}

Table 2. Lesions characteristics on the day of treatment.

\begin{tabular}{|l|c|}
\hline \multicolumn{1}{|c|}{ Lesions characteristics } & Values \\
\hline Severity of total treated lesions (\%) & \\
Grade I & $48(42.1)$ \\
Grade II & $66(57.9)$ \\
\hline
\end{tabular}




\subsection{Irradiances}

The measured irradiances over the 114 lesions ranged from $0.25 \mathrm{~mW} / \mathrm{cm}^{2}$ to $60 \mathrm{~mW} / \mathrm{cm}^{2}$ (average: $31.9 \mathrm{~mW} / \mathrm{cm}^{2}$; SD: $17.2 \mathrm{~mW} / \mathrm{cm}^{2}$ ). These values underline the above mentioned heterogeneous photoactivation over the treatment area provided by the Aktilite CL 128 (Figure 2).

\section{Irradiance}

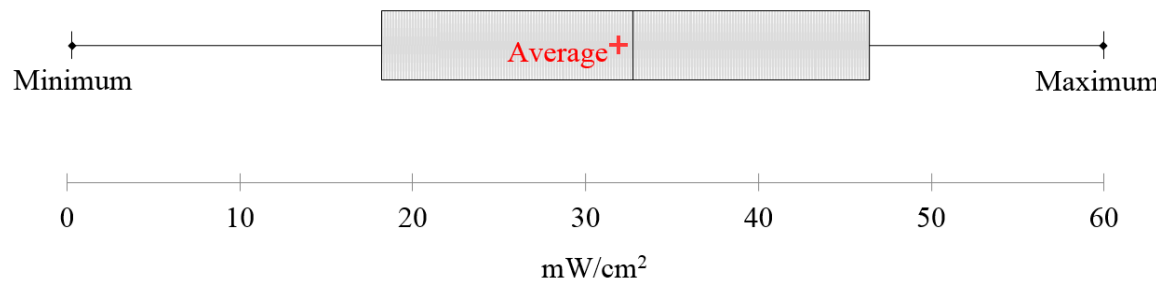

Figure 2. Box plot of irradiance.

\subsection{CR at 3 months}

Of the 114 AKs, 66 (57.9\%) were in CR at 3 months. There were 48 AKs (42.1\%) in incomplete response at three months.

\subsection{Effect of irradiance on the CR at 3 months}

The $66 \mathrm{AKs}$ in $\mathrm{CR}$ at 3 months received an average irradiance of $30.9 \mathrm{~mW} / \mathrm{cm}^{2}$ (standard deviation: $16.7 \mathrm{~mW} / \mathrm{cm}^{2}$; range: 2.7 to $60 \mathrm{~mW} / \mathrm{cm}^{2}$ ) while an average irradiance of $33.3 \mathrm{~mW} / \mathrm{cm}^{2}$ (standard deviation: $17.9 \mathrm{~mW} / \mathrm{cm}^{2} ; \mathrm{range}: 0.25$ to $60 \mathrm{~mW} / \mathrm{cm}^{2}$ ) was delivered to the $48 \mathrm{AKs}$ in incomplete response at 3 months. AKs in CR at 3 months had therefore not received higher irradiances than AKs in incomplete response at 3 months (Figure 3).

\section{Irradiance}

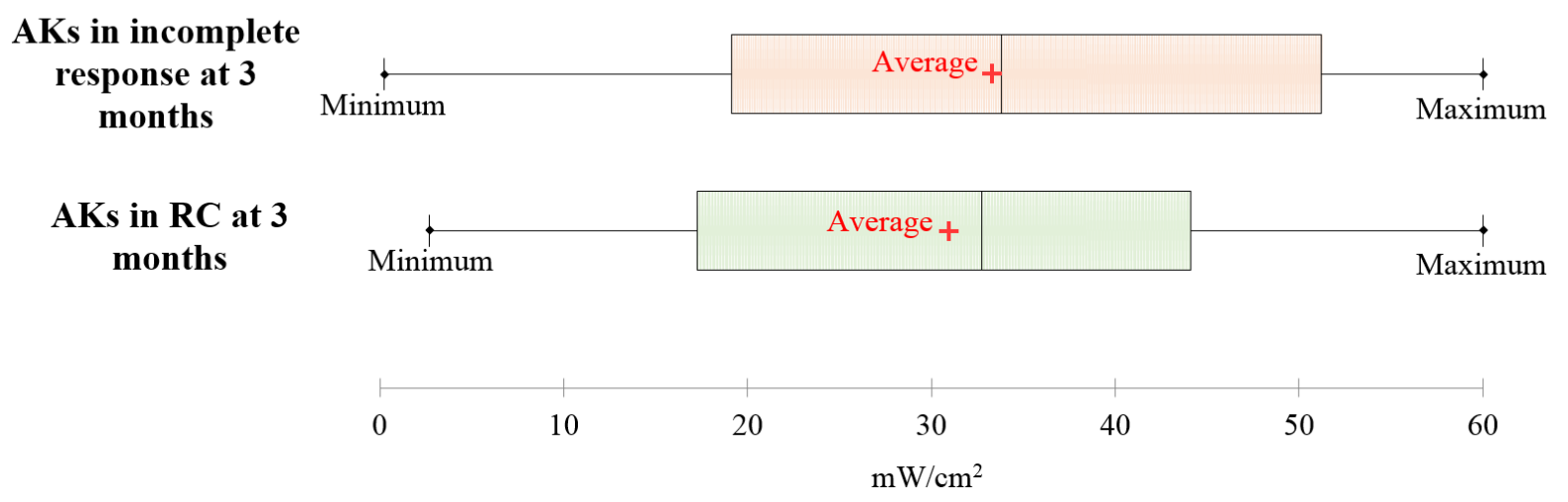

Figure 3. Box plots of irradiance for AKs in CR at 3 months (green plot) and AKs in incomplete response at 3 months (red plot). The crosses correspond to the average values, while the central vertical bars are the median ones. The left and right limits of the boxes are the first and third quartiles, respectively. The ends of the whiskers represent the minimum and maximum values. 
When graphically representing the rate of AKs in $\mathrm{CR}$ at 3 months according to irradiance, no effect was revealed (Figure 4). This no significant effect of irradiance on the CR at 3 months was confirmed by the statistical analysis (odds ratio for a $6 \mathrm{~mW} / \mathrm{cm}^{2}$-unit change, $0.96 ; 95 \%$ confidence interval, 0.83 to $1.10 ; \mathrm{p}=0.53$ ).

Due to this no effect, no minimum irradiance threshold for red-light photoactivation above which there is no further improvement in efficacy could be determined.

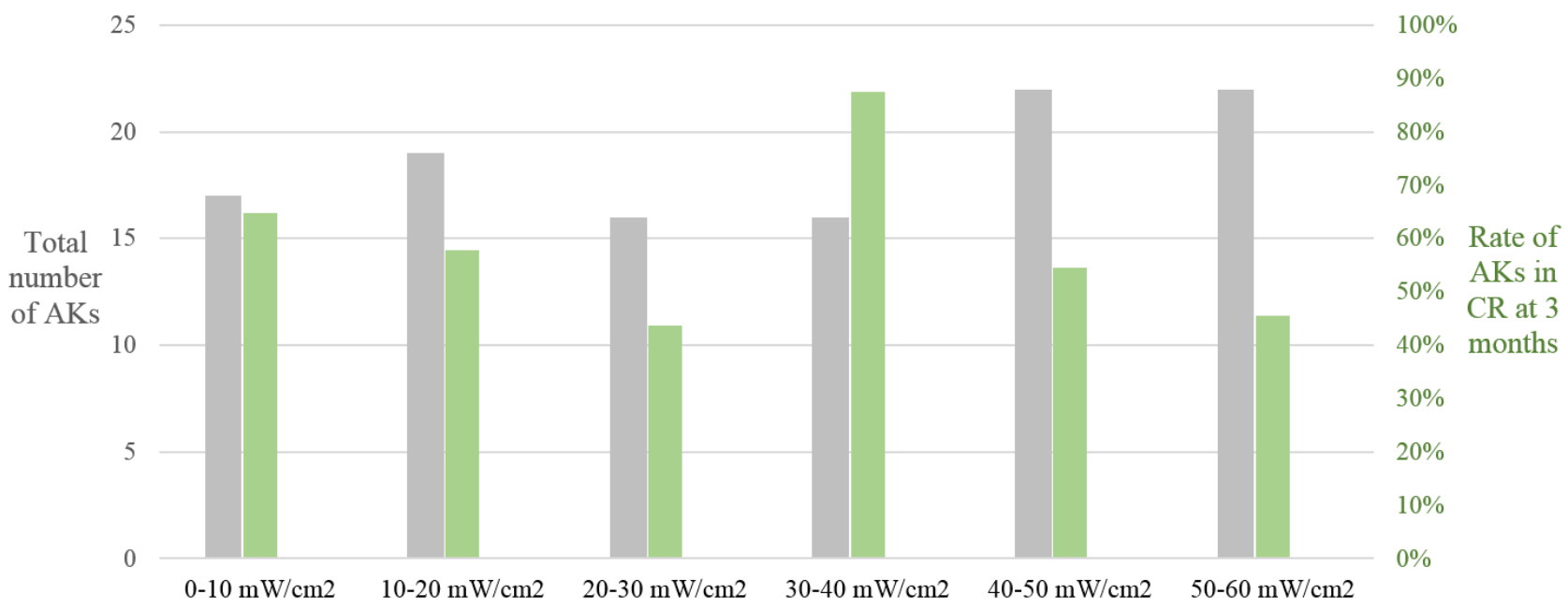

Figure 4. Number of AKs (grey bars) and rate of AKs in CR at 3 months (grey bars) according to the ranges of irradiances.

\section{DISCUSSION \& CONCLUSION}

In this study, the effect of the irradiance on CR at 3 months following C-PDT was demonstrated to be non-significant. This finding supports previous studies that have demonstrated similar efficacy for red-light photoactivation at irradiances of $15.4 \mathrm{~mW} / \mathrm{cm}^{2}$ [6], $12.3 \mathrm{~mW} / \mathrm{cm}^{2}$ [7] and $1.3 \mathrm{~mW} / \mathrm{cm}^{2}$ [8], respectively, compared to C-PDT.

Based on this finding, devices enabling red-light photoactivation at lower irradiance than that delivered by the Aktilite CL 128 should therefore provide similar efficacy than C-PDT.

The major benefit of red-light photoactivation at low irradiance is the reduction in pain. In fact, pain, which is the major drawback of C-PDT, has been shown to be an increasing function of irradiance ${ }^{[10,11]}$

The second benefit of red-light photoactivation at low irradiance is to allow compact light sources, consistent with ambulatory use.

Moreover, due to the use of a single irradiation time of 10 minutes for C-PDT in the present study, the no-effect finding can be extended to the light dose: the light dose has not effect on the CR at 3 months.

One limitation of the study is the low number of AKs and the subsequent limited statistical power. Further investigations involving many more AKs should be carried out to confirm the no significant effect of the irradiance on CR at 3 months.

The present study has been more widely addressed and discussed in a recent paper ${ }^{[12]}$. 


\section{REFERENCES}

[1] C. Morton, R. M. Szeimies, A. Sidoroff et al., "European Dermatology Forum Guidelines on topical photodynamic therapy," Eur J Dermatol, 25(4), 296-311 (2015).

[2] S. R. Wiegell, "Update on photodynamic treatment for actinic keratosis," Curr Probl Dermatol, 46, 122-8 (2015).

[3] D. Pariser, R. Loss, M. Jarratt et al., "Topical methyl-aminolevulinate photodynamic therapy using red lightemitting diode light for treatment of multiple actinic keratoses: A randomized, double-blind, placebo-controlled study," J Am Acad Dermatol, 59(4), 569-76 (2008).

[4] R. M. Szeimies, R. T. Matheson, S. A. Davis et al., "Topical methyl aminolevulinate photodynamic therapy using red light-emitting diode light for multiple actinic keratoses: a randomized study,” Dermatol Surg, 35(4), 586-92 (2009).

[5] S. R. Wiegell, M. Haedersdal, P. A. Philipsen et al., "Continuous activation of PpIX by daylight is as effective as and less painful than conventional photodynamic therapy for actinic keratoses; a randomized, controlled, single-blinded study,” Br J Dermatol, 158(4), 740-6 (2008).

[6] P. Gholam, I. Bosselmann, A. H. Enk et al., "Low irradiance compared with conventional photodynamic therapy in the treatment of actinic keratoses," Photodermatol Photoimmunol Photomed, (2018).

[7] C. Vicentini, A. S. Vignion-Dewalle, E. Thecua et al., "Photodynamic therapy for actinic keratosis of the forehead and scalp: a randomized, controlled, phase II clinical study evaluating the noninferiority of a new protocol involving irradiation with a light-emitting, fabric-based device (the Flexitheralight protocol) compared with the conventional protocol involving irradiation with the Aktilite CL 128 lamp," Br J Dermatol, (2018).

[8] S. Mordon, A. S. Vignion-Dewalle, H. Abi-Rached et al., "The conventional protocol versus a protocol including illumination with a fabric-based biophotonic device (the Phosistos protocol) in photodynamic therapy for actinic keratosis: a randomized, controlled, non-inferiority clinical study," Br J Dermatol, (2019).

[9] F. Lecomte, A. S. Vignion-Dewalle, C. Vicentini et al., "Evaluating the Noninferiority of a New Photodynamic Therapy (Flexitheralight) Compared With Conventional Treatment for Actinic Keratosis: Protocol for a Phase 2 Study," JMIR Res Protoc, 8(4), e11530 (2019).

[10] Z. Apalla, E. Sotiriou, D. Panagiotidou et al., "The impact of different fluence rates on pain and clinical outcome in patients with actinic keratoses treated with photodynamic therapy," Photodermatol Photoimmunol Photomed, 27(4), 181-5 (2011).

[11] S. R. Wiegell, J. Skiveren, P. A. Philipsen et al., "Pain during photodynamic therapy is associated with protoporphyrin IX fluorescence and fluence rate,” Br J Dermatol, 158(4), 727-33 (2008).

[12] C. Vicentini, A. S. Vignion-Dewalle, E. Thecua et al., "Photodynamic therapy for actinic keratosis of the forehead and scalp with the Aktilite CL 128: Is there a cut-off value for PpIX-weighted irradiance for effective treatment?," Photodermatol Photoimmunol Photomed, (2019). 